\title{
COMPARISON OF TURBULENCE INTEGRAL LENGTH SCALE DETERMINATION METHODS
}

\author{
ARSENII TRUSH ${ }^{1}$, STANISLAV POSPÍŠIL ${ }^{1} \&$ HRVOJE KOZMAR $^{2}$ \\ ${ }^{1}$ Institute of Theoretical and Applied Mechanics of the Czech Academy of Sciences, Czech Republic \\ ${ }^{2}$ Faculty of Mechanical Engineering and Naval Architecture, University of Zagreb, Croatia
}

\begin{abstract}
Grids at the inlet of the wind-tunnel test section have been commonly used to create turbulent conditions for model experiments concerning civil engineering applications. The parameters of the generated turbulence depend on the geometry of the grid and the distance from the grid to the measurement position. This work presents the experimental results of the grid-generated turbulence and a comparative analysis of an applicability of the von Kármán and Fichtl-McVehil autospectra models and autocorrelation methods (zero-crossing and exponential) for calculation of integral turbulence length scales. A square mesh array of rectangular bars was tested in a closed-circuit low velocity wind tunnel. The initial free stream turbulence was $I_{u}=0.75 \%$. The measurements were carried out using the $\mathrm{X}$-wire CTA probe installed at distances ranging from $1.35 \mathrm{~m}$ to $8.4 \mathrm{~m}$ downstream of the tested grid at velocities from $1.5 \mathrm{~m} / \mathrm{s}$ to $9 \mathrm{~m} / \mathrm{s}$. The fitting suitability of various spectral models depends on the inflow velocity and turbulence intensity. Due to a greater flexibility and quantity of fitting coefficients, the Fichtl-McVehil model fits better in the area around the spectral peak at low flow velocity up to $3.4 \mathrm{~m} / \mathrm{s}$, while von Kármán models more accurately represent the measured spectrum at higher flow velocities. The methods based on using the correlation coefficients are equally suitable for flows at all measured velocities and turbulence intensities. The exponential method yields more stable distance-toscale characteristics with smaller deviations.
\end{abstract}

Keywords: turbulence, integral turbulence length scale, calculation methods, wind-tunnel experiments.

\section{INTRODUCTION}

Wind flow and turbulence in the atmospheric boundary layer substantially affect static and dynamic loads on engineering structures [1], cause buffeting and other aeroelastic effects on slender structures [2], affect natural ventilation and air pollution in open and urban areas [3]. The influence of atmospheric turbulence is also important when evaluating the economic efficiency of wind farms, e.g. [4]. Turbulence intensity and integral turbulence length scale are important characteristics of turbulence and they have been commonly used in engineering practice [5]-[7], whereas it is necessary to ensure a compliance and an applicability of the results obtained using various methods. A particular attention needs to be paid to the smallscale experiments in wind tunnels [8]. The focus of the present study is a comparison of the integral turbulence length scales calculated using various methods, whereas the flow and turbulence characteristics in a boundary layer wind tunnel are created using a grid placed at the test-section inlet.

The absolute velocity $\left(u_{x}, u_{y}, u_{z}\right)$ is a sum of the mean velocities $\bar{u}_{x}, \bar{u}_{y}, \bar{u}_{z}$ and the fluctuating components $u_{x}{ }^{\prime}, u_{y}{ }^{\prime}, u_{z}{ }^{\prime}$, which procedure is known as Reynolds averaging:

- $\quad x$ - longitudinal direction $u_{x}=\bar{u}_{x}+u_{x}{ }^{\prime}$;

- $y$ - lateral direction $u_{y}=\bar{u}_{y}+u_{y}{ }^{\prime}$;

- $\quad z$ - vertical direction $u_{z}=\bar{u}_{z}+u_{z}{ }^{\prime}$;

Turbulent velocity fluctuations may be considered as a result of the eddies that are being transferred by the mean flow velocity. Each eddy is characterized by a periodic motion of the circular frequency $\omega=2 \pi f$, where $f$ is the frequency, or equivalently by a wave number 
$k_{w}=2 \pi / \lambda$, where $\lambda$ is the wave length. $L_{u, x}, L_{u, y}$ and $L_{u, z}$ integral turbulence length scales represent the average size of vortices containing the highest energy in the respective directions, i.e. longitudinal, lateral and vertical directions, caused by the wind velocity fluctuations in the longitudinal direction. Large eddies eventually dissipate in small eddies along with the turbulence kinetic energy (TKE) transfer from large to small eddies - this process is known as the energy cascade. The area of the highest frequencies and the smallest eddies is known as the dissipation zone. In that area, the transformation of the TKE into heat is a dominant phenomenon. The dissipation zone is usually not relevant for wind engineering applications. The maximum energy $S_{u}$ is contained in the middle of the spectrum inertial subrange, where the dominant forces are the inertial forces. In that area the Kolmogorov law is valid

$$
S_{u}(f) \approx k_{w}^{-\frac{5}{3}},
$$

where $k_{w}=$ the wave number of the eddy.

The distribution of turbulence fluctuations in time as a zero-mean random process is described by the spectral density function, or in other words - power spectrum

$$
S_{u, x}\left(x_{1}, x_{2}, f\right)=\int_{-\infty}^{+\infty} \lim _{t_{0} \rightarrow \infty} \frac{1}{2 t_{0}} \int_{-t_{0}}^{t_{0}} u\left(x_{1}, t\right) u\left(x_{2}, t+\Delta t\right) e^{-i 2 \pi f \Delta t} d t d \Delta t,
$$

where:

$u(x)=$ wind velocity in point $x$;

indices 1 and 2 denote two different positions in space;

$\Delta x=$ distance between points $x_{1}$ and $x_{2}$;

$\Delta t=$ time shift.

A schematic view of the velocity power spectrum is given in Fig. 1.

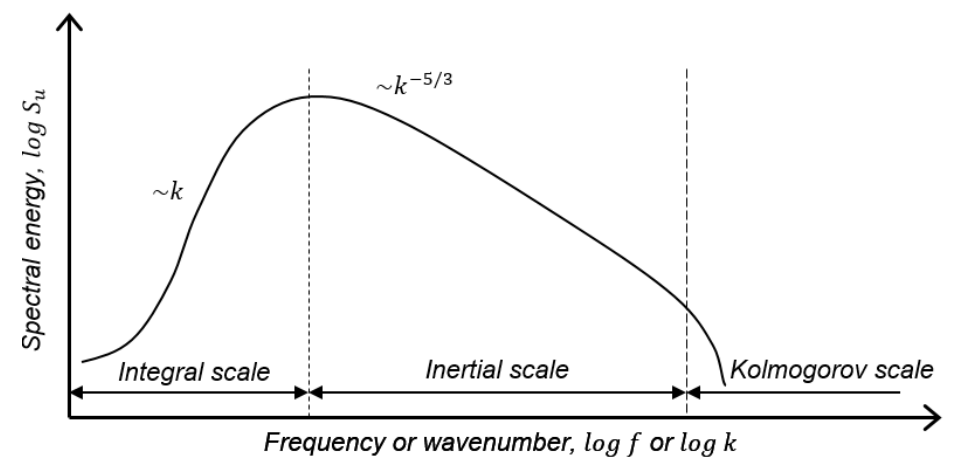

Figure 1: Schematic representation of the power spectral density of longitudinal velocity fluctuations [9].

The contribution to the mean velocity variance (or square of the standard deviation) in the range of frequencies from $f$ to $f+d f$, is given by $S u(f) \cdot d f$, where $S u(f)$ is the spectral density function of $u(t)$. Then integrating over all frequencies

$$
\sigma_{u}^{2}=\int_{0}^{\infty} S_{u}(f) d f
$$

where $\sigma_{u}=$ the standard deviation of the longitudinal wind velocity component $u$. 
In eqn (3), the integral over time is a corresponding spatial correlation function for velocity fluctuations

$$
R_{u, x}(\Delta x)=\int_{-t_{0}}^{t_{0}} u\left(x_{1}, t\right) u\left(x_{2}, t+\Delta t\right) e^{-i 2 \pi f \Delta t} d t d \Delta t .
$$

According to Sockell [10], $L_{u, x}$ is given by

$$
\begin{gathered}
L_{u, x}=\int_{0}^{\infty} R_{u, x}(x+\Delta x) d \Delta x, \\
R_{u, x}(\Delta x)=\frac{{u^{\prime}}_{1}(t) \cdot u^{\prime}{ }_{2}(t)}{\sqrt{u^{\prime 2}} \cdot \sqrt{u_{2}^{2}}},
\end{gathered}
$$

where the indices 1 and 2 denote two different positions in space.

One of the most notable assumptions concerning turbulence is the Taylor frozen turbulence hypothesis [11] suggesting that in a single-phase flow, turbulent vortices are transported by the mean velocity of the main flow. Under the assumption of the Taylor frozen turbulence hypothesis, the autocorrelation function provided in eqn (6) may be also calculated based on the time history at one point only

$$
R_{u, x}(\Delta x)=\frac{u_{1}^{\prime}(t) \cdot u_{1}^{\prime}(t-\Delta t)}{u_{1}^{\prime 2}}=R_{u, x}(\Delta t) .
$$

The integral length scale could be accordingly calculated as

$$
L_{u, x}=u \int_{0}^{\infty} R_{u, x}(t) d \Delta t
$$

where the time period $\Delta t$ is

$$
\Delta t=\frac{\Delta x}{u} .
$$

This hypothesis has been confirmed by many experimental studies performed in a singlephase flow downstream of a turbulence-generation grids and with some corrections in developed natural turbulent flows, e.g. [12].

\section{METHODS OF DETERMINATION OF THE INTEGRAL TURBULENCE LENGTH SCALES}

The integral turbulence length scales can be calculated in various ways, based on physically different interpretations. When providing the results of the turbulence integral time and integral turbulence length scales, it is commonly necessary to indicate the method according to which these values were calculated.

\subsection{Methods based on the spatial-temporal correlation of turbulent structures}

- Auto-(cross-) correlation zero-crossing method refers to correlated turbulence structures: in this case, the integral time scale is obtained by using the correlation coefficients, e.g. eqn (7), as they decrease to zero. Consequently, based on the Taylor hypothesis, the time scale is used to determine the integral turbulence length scale.

- Exponential method: It is similar to the zero-crossing method; however, in this case, the time period needed for the correlation curve to decrease to $1 / \mathrm{e}$ is used as the integral time scale. It facilitates a possibility to avoid the issues that may occur in case the correlation function does not cross the horizontal axis due to random fluctuations, e.g. [13]. 


\subsection{Auto-power spectral methods}

In the engineering practice, the power auto-spectral density (power auto-spectrum) has been commonly used for dynamic analysis in the frequency domain of structures experiencing aerodynamic loads, but also to determine the integral turbulence length scales. Spectral turbulence models are mostly based on (semi-) empirical data. The auto-spectrum and the auto-correlation function are interrelated by the Fourier transform pairs as it is known from the Wiener-Khintchine relations. Spectral techniques define the integral turbulence length scale as the vortex size corresponding to the maximum spectral energy. In order to determine the length scale, the time scale corresponding to the frequency with the maximum energy in the spectrum (i.e. peak frequency) is used. The peak frequency is obtained from the fitting of spectral turbulence models to the measured turbulence spectrum.

2.2.1 Von Kármán turbulence autospectrum model

One of the first methods was proposed by von Kármán [14] as a statistical interpretation of the experimental data obtained in a wind tunnel

$$
\frac{f \cdot S_{u}(f)}{\sigma_{u}^{2}}=\frac{4 f_{L}}{\left(1+70.78 \cdot f_{L}^{2}\right)^{\frac{5}{6}}},
$$

where:

$f=$ frequency;

$f_{L}=$ dimensionless coordinate

$$
f_{L}=\frac{f \cdot L_{u, x}(z)}{u(z)}
$$

When the frequency approaches zero, the integral turbulence length scale, after differentiating eqn (10), can be obtained from the peak frequency

$$
L_{u, x}=\frac{\sqrt{3 / 2} \cdot f_{L}}{\sqrt{70.78}}=\frac{0.146 \cdot u(z)}{f_{\text {peak }}},
$$

where $f_{\text {peak }}=$ frequency of the maximum $f \cdot S u(f)$ value.

Until now, this is one of the most widely used power spectra models used in laboratory studies. Its application in practice is limited to the safety design of engineering structures characterized by the low damping and low structure-to-flow mass relation. It is also used in some regulatory documents, e.g. [15], [16].

2.2.2 Fichtl-McVehil turbulence autospectrum model

Another spectral model was presented by Fichtl and McVehil [17] as

$$
\frac{f \cdot S_{u}(f)}{\sigma_{u}^{2}}=\frac{A_{j} f_{L}}{\left(1+B_{j} \cdot f^{\gamma_{j}}\right)^{5 / 3 \gamma_{j}}} .
$$

The fitting parameters $A_{j}, B_{j}, \gamma_{j}$ are defined for the specific turbulence conditions. In case of $A_{j}=4 ; B_{j}=70.8 ; \gamma_{j}=2$, this model is similar to von Kármán model.

\section{EXPERIMENTAL SETUP}

Experiments were carried out in a climatic boundary-layer wind tunnel of the Institute of Theoretical and Applied Mechanics in Czech Republic. The rectangular cross section of the aerodynamic test section is $1.9 \mathrm{~m}$ wide and $1.8 \mathrm{~m}$ high and equipped with a turntable used for placing structural models. The test-section length is $11.0 \mathrm{~m}$. The turbulence intensity at 
the test-section inlet is $I_{u}=0.75 \%$. The turbulent flow was generated by square mesh grid of rectangular bars, (see Fig. 2), which creates nearly isotropic turbulence, e.g. [18]. The distance between the bars was $180 \mathrm{~mm}$, and the bars were $55 \mathrm{~mm}$ wide and $25 \mathrm{~mm}$ thick. Multichannel CTA system by Dantec Dynamics with one X-wire CTA probe was used to measure velocity time series.

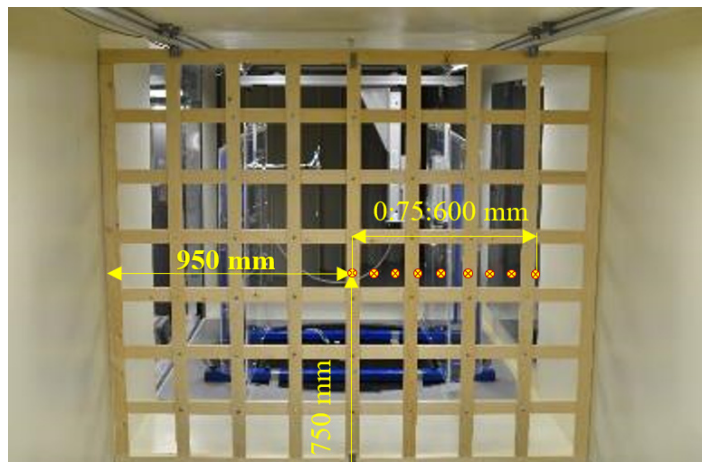

Figure 2: Wooden grid used for turbulence generation.

Measurements were taken at the distances of 1.35, 2.1, 3.85, 4.6, 5.1, 5.9, 7.1 and $8.4 \mathrm{~m}$ downstream of the grid. The profile of the flow velocity was measured in 9 points at the height of $750 \mathrm{~mm}$ above the wind-tunnel floor with $75 \mathrm{~mm}$ horizontal lateral step from the center of the cross section (see Fig. 2). The time record length was $60 \mathrm{~s}$ at the $1,000 \mathrm{kHz}$ acquisition rate.

\section{RESULTS}

\subsection{Turbulence intensity}

The turbulence intensity at various distances downstream of the grid is shown in Fig. 3.

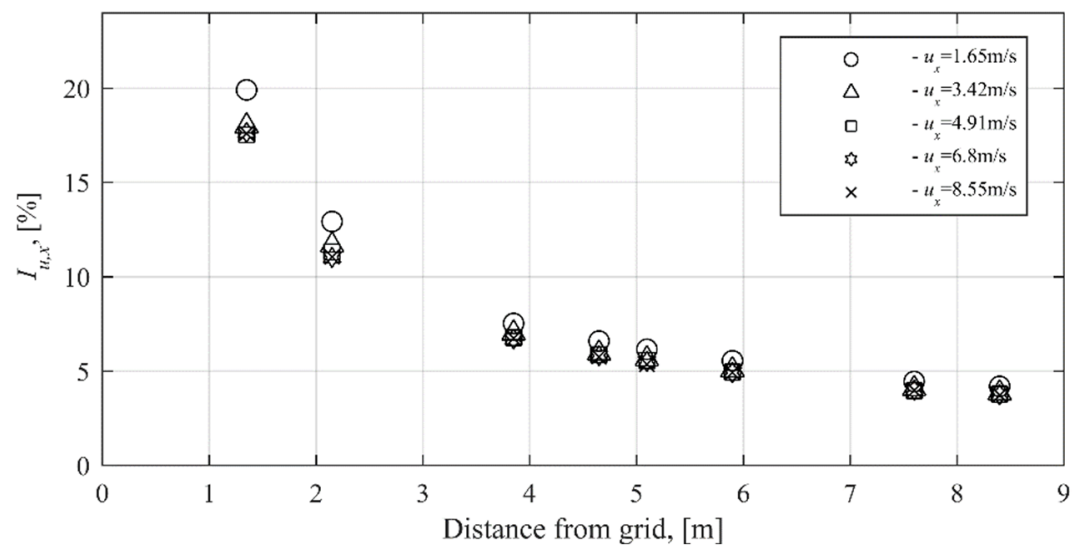

Figure 3: Experimentally obtained turbulence intensity at various distances downstream of the grid. 
It can be observed that the recorded values obtained for the flow at lower velocity are slightly larger immediately downstream of the grid. With increasing the downstream distance from the grid, the difference between the turbulence intensity recorded at various flow velocities decreases.

\subsection{Von Kármán spectral model}

To determine the peak spectral frequency, the normalized power spectra were fitted using the nonlinear least-square method. To simplify and speed up the fitting procedure, the experimental dataset was divided into 10 -second blocks with the $50 \%$ overlap filtered using the rectangular window. Consequently, the average value of the measured spectra was adopted as the final power spectrum (Fig. 5). The values calculated according to Roach [18] were used as a reference. It should be noted that this methodology proved to be applicable for square rods, while the rectangular rods were used in the present study.

The overestimated values of the integral turbulence length scales at lower velocities are likely due to the not completely adequate fitting of the model to the measured spectrum in the high frequency range (Fig. 4(a)). Due to the lower TKE input, the dissipation is enhanced and the Kolmogorov range shifts to lower frequencies. At higher flow velocities, i.e. higher TKE input, the Kolmogorov range is outside the analyzed frequency range and the fitting indicates less deviations (Fig. 4(b)).

The scatter of values along the profile at all tested velocities is negligible (Fig. 6).
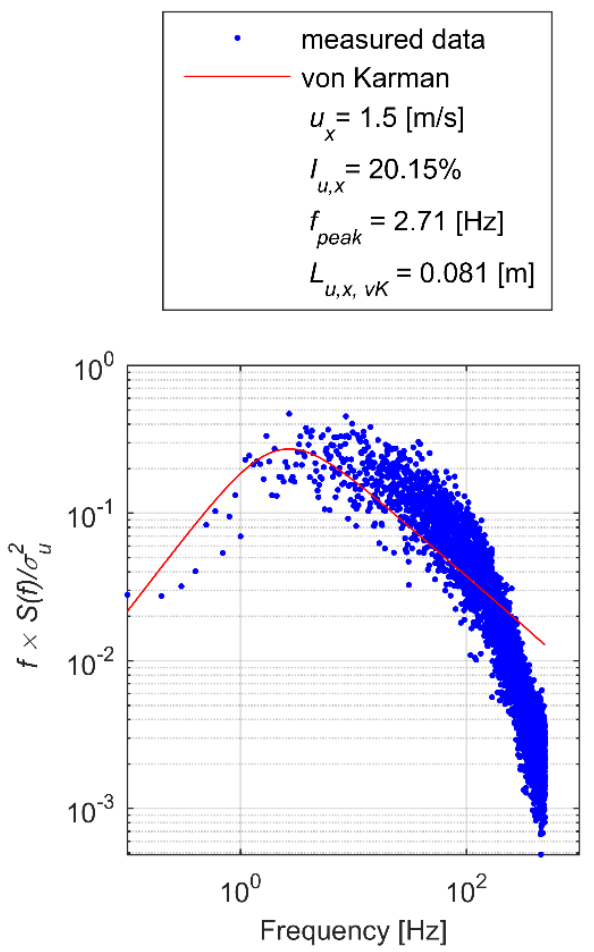

(a)
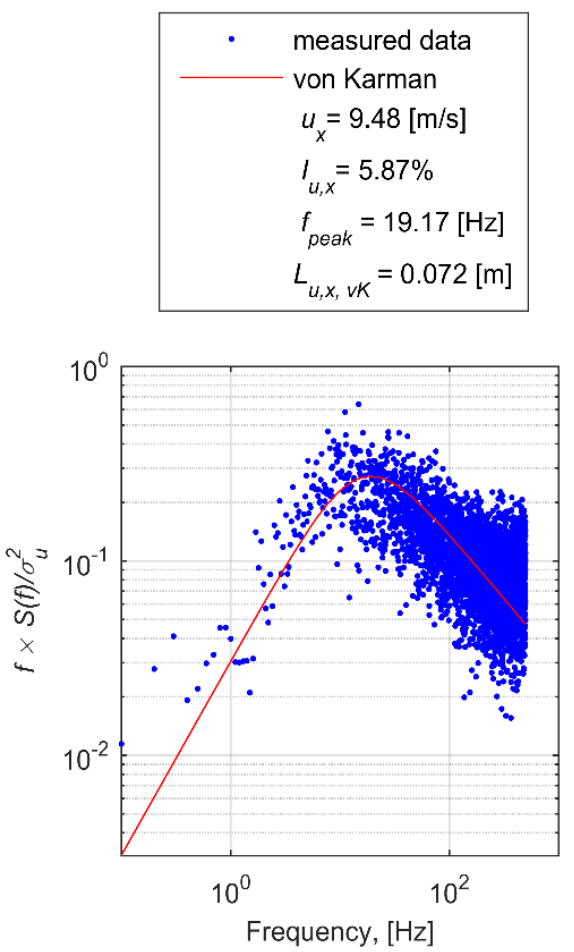

(b)

Figure 4: Fitting of von Kármán model to the measured power spectrum at the downstream distance of (a) $1.35 \mathrm{~m}$; and (b) $3.85 \mathrm{~m}$. 


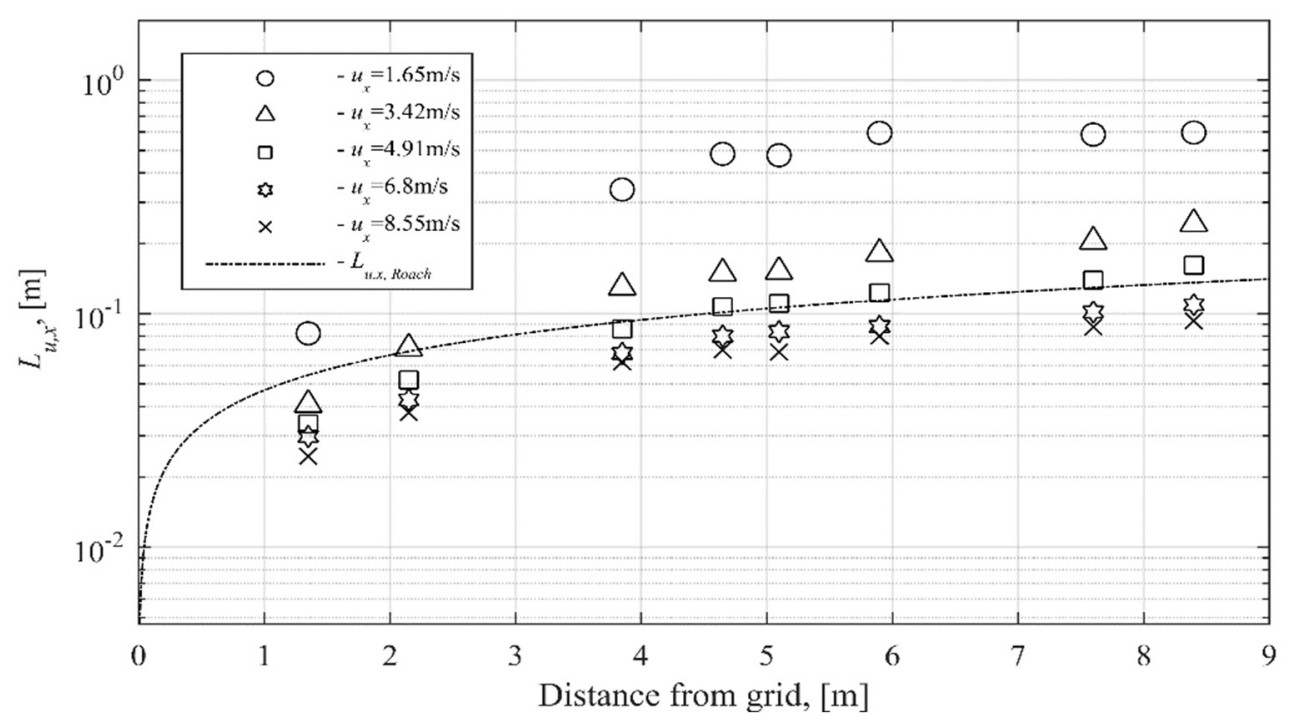

Figure 5: Integral turbulence length scales obtained by von Kármán model fitting.

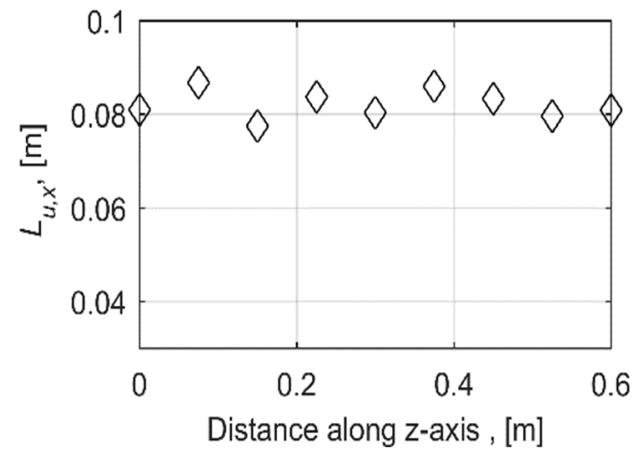

(a)

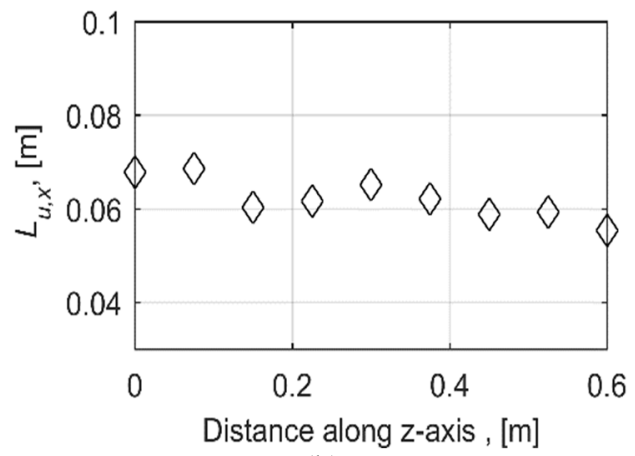

(b)

Figure 6: Distribution of integral turbulence length scales obtained by von Kármán spectral model fitting at (a) The downstream distance of $1.35 \mathrm{~m}$ and $u_{x}=1.65$ $\mathrm{m} / \mathrm{s}$; and (b) The downstream distance of $3.85 \mathrm{~m}$ and $u_{x}=8.55 \mathrm{~m} / \mathrm{s}$.

\subsection{Fichtl-McVehil spectral model}

The results obtained using the Fichtl-McVehil model are presented in Fig. 7. This model also does not provide an additional explanation for the Kolmogorov range but has more coefficients that enables better fitting for larger vortices. This fact allows for reducing the shift of the peak frequency and the respective error in the integral turbulence length scale estimation (Fig. 8). At the same time, at higher velocities and especially at high turbulence, the flexibility of the fitting coefficients may yield inaccuracies, which behavior is similar to von Kármán model at low flow velocities. 


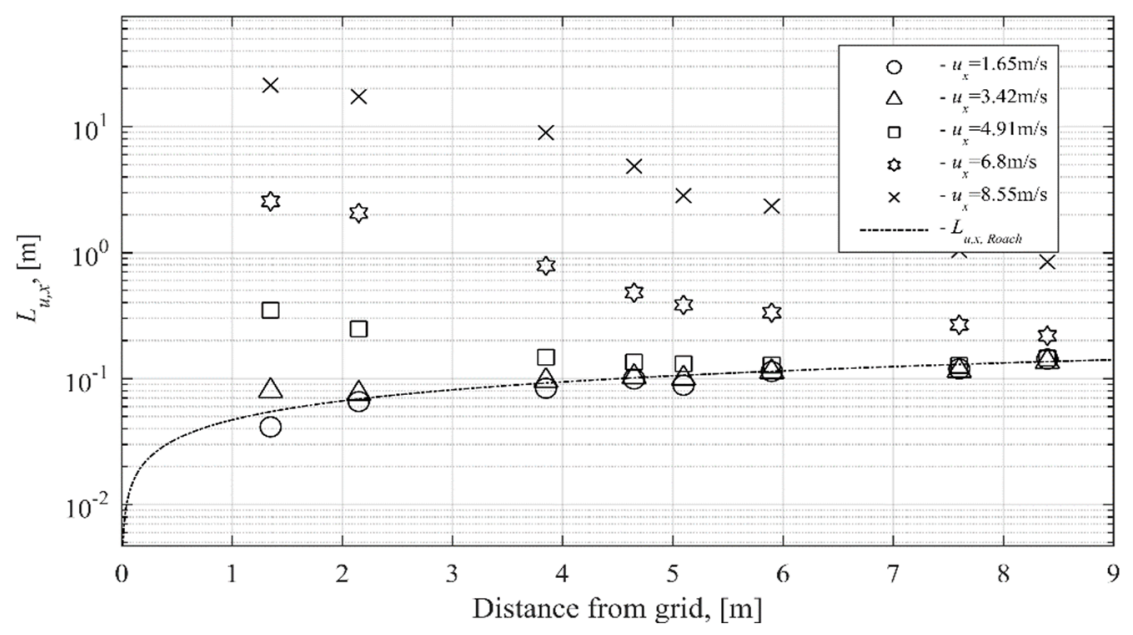

Figure 7: Integral turbulence length scales obtained by fitting of Fichtl-McVehil model.
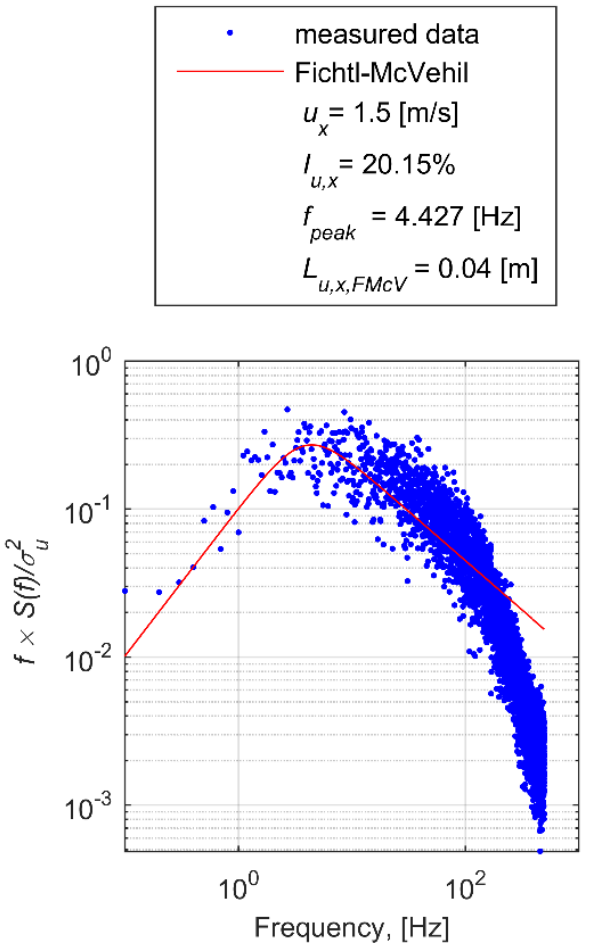

(a)
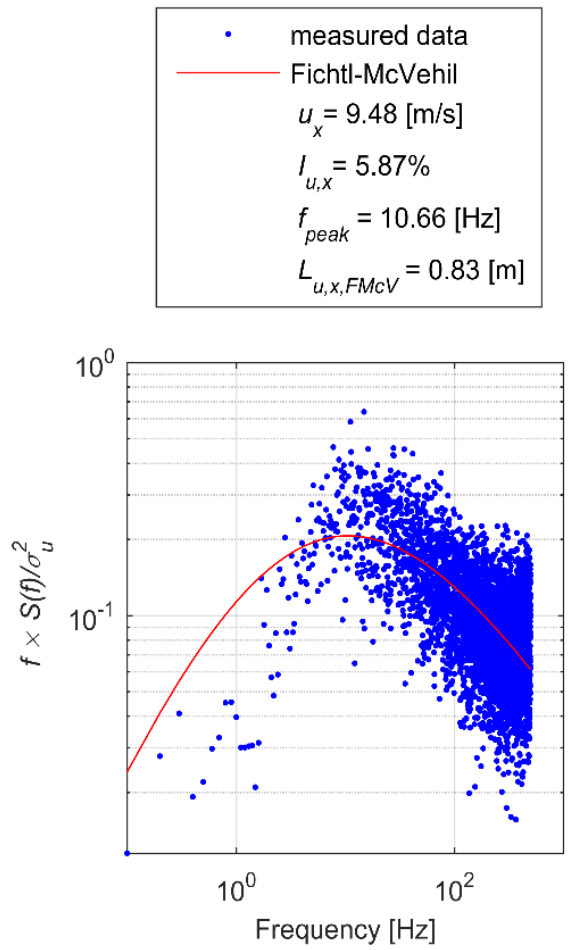

(b)

Figure 8: Fitting of Fichtl-McVehil model to the measured power spectrum at the downstream distance of (a) $1.35 \mathrm{~m}$; and (b) $3.85 \mathrm{~m}$. 


\subsection{Auto-correlation methods}

The turbulence integral length scales obtained by using the zero-crossing and exponential methods are presented in Figs 9 and 10, respectively. An example of the correlation graph is shown in Fig. 11. It may be observed that the values obtained by using the zero-crossing method have larger dispersion at various mean flow velocities at various positions along the test section (Fig. 12). The exponential method gives slightly lower values of the integral turbulence length scales compared to the zero-crossing method.

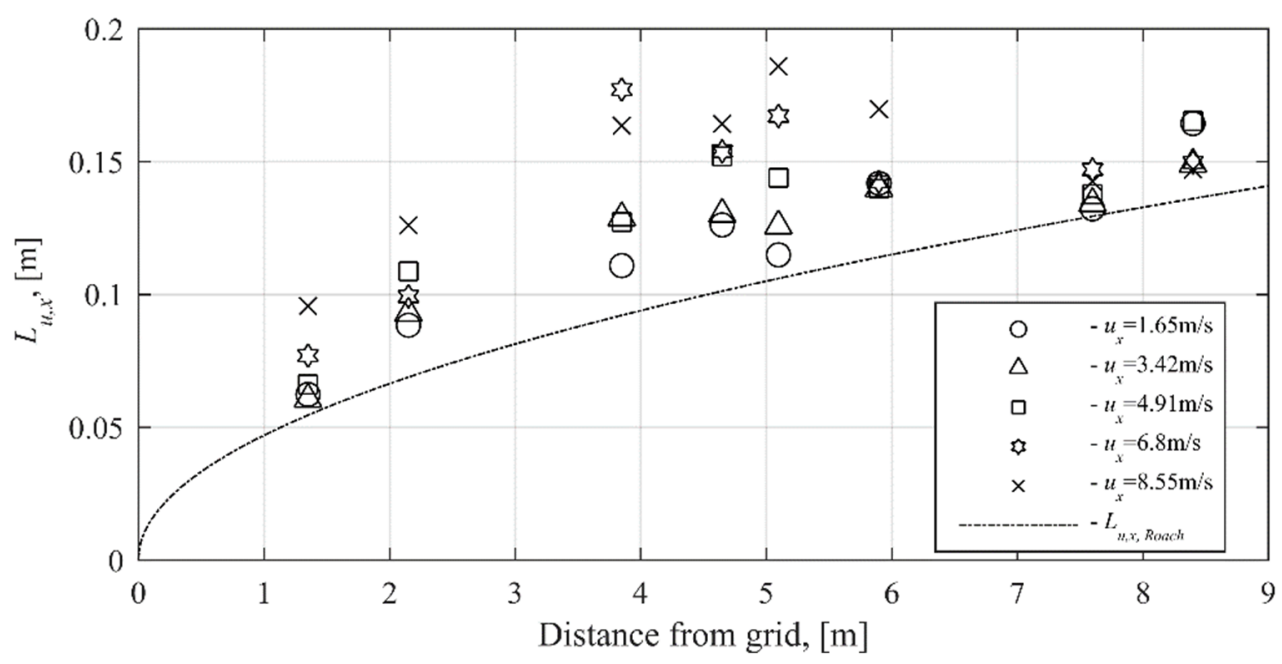

Figure 9: Integral turbulence length scales calculated by using the zero-crossing method at various distances downstream of the grid.

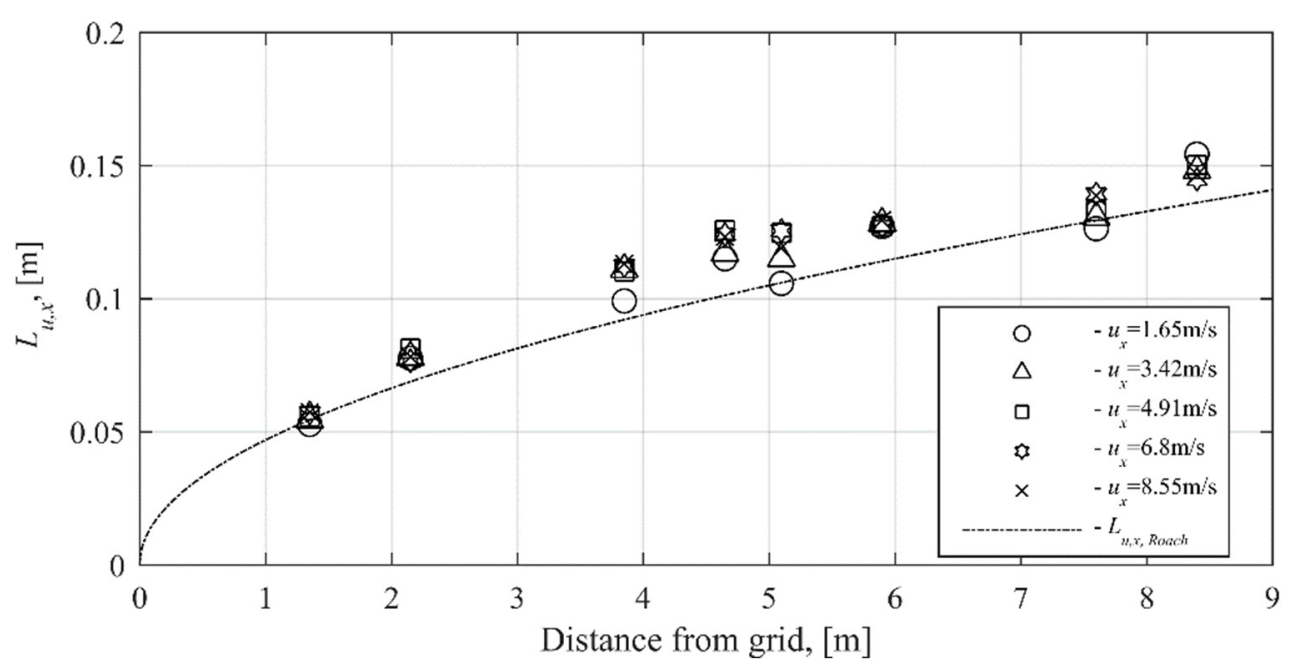

Figure 10: Integral turbulence length scales calculated by using the exponential method at various distances downstream of the grid. 


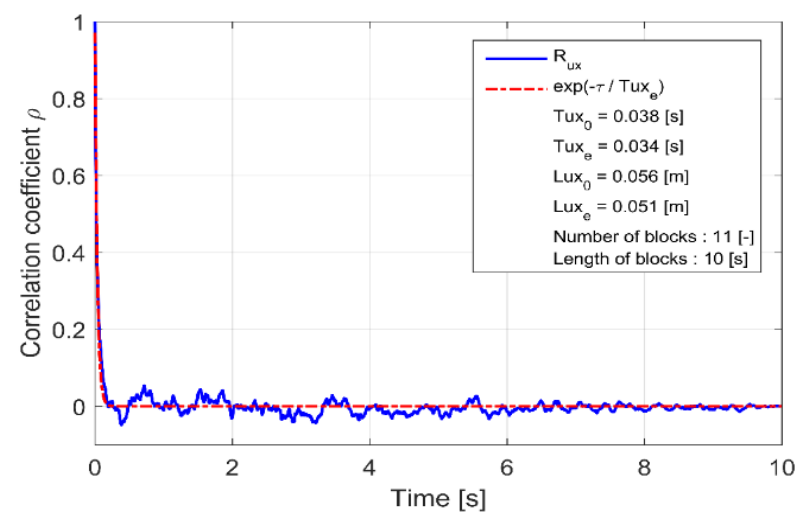

Figure 11: Determination of integral turbulence length scales by using the zero-crossing and exponential decay methods.

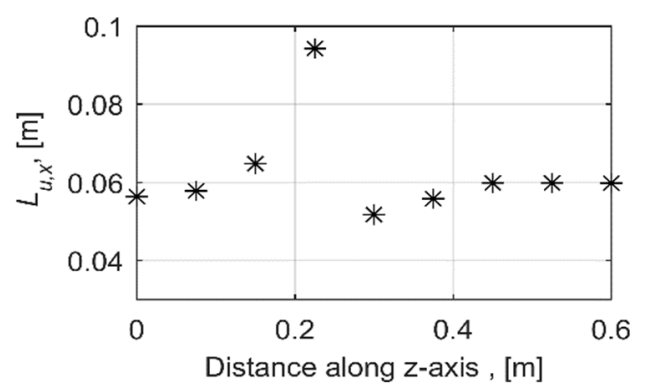

(a)

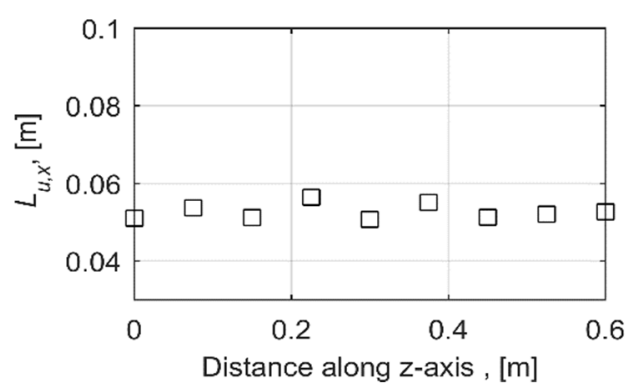

(b)

Figure 12: Distribution of integral turbulence length scales obtained at the downstream distance of $1.35 \mathrm{~m}$ and $u_{\mathrm{x}}=1.65 \mathrm{~m} / \mathrm{s}$ by applying the (a) Zero-crossing; and (b) Exponential methods.

\section{CONCLUSIONS}

A preliminary analysis of the applicability of various methods to determine integral turbulence length scales in the grid-generated turbulence was presented. In the analyzed frequency range, the autospectra curves differ from the measured spectrum because of the lower TKE input at low flow velocities. Because of a greater flexibility and more fitting coefficients, at low flow velocities the Fichtl-McVehil model fits better to the experimental results at frequencies around the spectral peak. On the other hand, von Kármán model more accurately corresponds to the measured spectrum at moderate and high flow velocities. Improvements may possibly be achieved by limiting the analyzed frequency range and further adjusting the model coefficients (for the Fichtl-McVehil model). Another issue with those spectral models is a necessity of visual control, which becomes rather difficult when analyzing a large amount of data. The methods based on using the covariance coefficients proved to be equally suitable at studied flow velocities and turbulence. The exponential method seems to be more stable with smaller deviations. For this reason, the exponential method is recommended concerning the repeatability. 


\section{ACKNOWLEDGEMENTS}

The research grant No. 19-04695S of the Czech Science Foundation (GAČR) is appreciated. The authors also acknowledge a partial funding of the Croatian Science Foundation IP-201606-2017 (WESLO).

\section{REFERENCES}

[1] Morrison, M.J. \& Kopp, G.A., Effects of turbulence intensity and scale on surface pressure fluctuations on the roof of a low-rise building in the atmospheric boundary layer. J. of Wind Engineering and Industrial Aerodynamics, 183, pp. 140-151, 2018.

[2] Chen, X. \& Kareem, A., Nonlinear response analysis of long-span bridges under turbulent winds. Journal of Wind Engineering and Industrial Aerodynamics, 89, pp. 1335-1350, 2001.

[3] Petäjä, T. et al., Enhanced air pollution via aerosol-boundary layer feedback in China. Scientifical Reports, 6, 2016.

[4] Optis, M. \& Perr-Sauer, J., The importance of atmospheric turbulence and stability in machine-learning models of wind farm power production. Renewable and Sustainable Energy Reviews, 112, pp. 27-41, 2019.

[5] Fenerci, A., Øiseth, O. \& Rønnquist, A., Long-term monitoring of wind field characteristics and dynamic response of a long-span suspension bridge in complex terrain. Engineering Structures, 147, pp. 269-284, 2017.

[6] Fernando Bastos, F., Elsa Caetano, E., Cunha, Á., Cespedes, X. \& Flamand, O., Characterisation of the wind properties in the Grande Ravine viaduct. Journal of Wind Engineering and Industrial Aerodynamics, 173, pp. 112-131, 2018.

[7] Diana, G. et al., Comparisons between wind tunnel tests on a full aeroelastic model of the proposed bridge over Stretto di Messina and numerical results. Journal of Wind Engineering and Industrial Aerodynamics, 54-55, pp. 101-113,1995.

[8] Vita, G., Hemida, H., Andrianne, T. \& Baniotopoulos, C.C., Generating atmospheric turbulence using passive grids in an expansion test section of a wind tunnel. Journal of Wind Engineering and Industrial Aerodynamics, 178, pp. 91-104, 2018.

[9] Yang, Q., Tian, Y., Li, B. \& Chen, B., Statistical spectrum model of wind velocity at Beijing Meteorological Tower. Proceedings of 7th International Colloquium on Bluff Body Aerodynamics and Applications (BBAA7), 2012.

[10] Sockell, H., Aerodynamik der Bauwerke, Vieweg: Braunschweig, Wiesbaden, 1984.

[11] Hill, R., Corrections to Taylor's frozen turbulence approximation. Atmospheric Research, 40(2-4), pp. 153-175, 1996.

[12] Batchelor, G., The Theory of Homogeneous Turbulence, Cambridge University Press: London, 1953.

[13] Flay, R. \& Stevenson, D., Integral length scales in atmospheric boundary-layer near the ground. Proceedings of the 9th Australasian Fluid Mechanics Conference, 1986.

[14] Von Kármán, T., Progress in the statistical theory of turbulence. Proc. Natl. Acad. Sci. United States Am., 34(11), pp. 530-539, 1948.

[15] AIJ-RLB, Recommendations on Loads for Buildings, Architectural Institute of Japan: Japan, 2004.

[16] AS/NZS 1170.2-2002, Structural design actions: Wind actions, 2002.

[17] Fichtl, G.H. \& McVehil, G., Longitudinal and lateral spectra of turbulence in the atmospheric boundary layer at the Kennedy Space Center. Journal of Applied Meteorology, 9, pp. 51-63, 1970.

[18] Roach, P., The generation of nearly isotropic turbulence by means of grids. International Journal of Heat and Fluid Flow, 8(2), 1987. 